\title{
Primary Sjögren's Syndrome Adversely Affects the Female Sexual Function Assessed by the Female Sexual Function Index: A Case-Control Study
}

\author{
Çağlar YILDIZ, ${ }^{1}$ Savaş KARAKUŞ, ${ }^{1}$ Özlem BOZOKLU AKKAR, ${ }^{1}$ Ali ŞAHİN, ${ }^{2}$ \\ Birkan BOZKURT, ${ }^{3}$ Ali YANIK ${ }^{1}$ \\ ${ }^{1}$ Department of Gynecology and Obstetrics, Medical Faculty of Cumhuriyet University, Sivas, Turkey \\ ${ }^{2}$ Department of Internal Medicine, Division of Rheumatology, Medical Faculty of Cumhuriyet University, Sivas, Turkey \\ ${ }^{3}$ Department of General Surgery, Medical Faculty of Cumhuriyet University, Sivas, Turkey
}

\begin{abstract}
Objectives: This study aims to evaluate the sexual function of females with primary Sjögren's syndrome (pSS) in comparison to healthy females by using the Female Sexual Function Index (FSFI).

Patients and methods: This case-control study included 31 female patients (mean age $46.0 \pm 10.2$ years; range 30 to 68 years) with pSS and 27 healthy females (mean age $44.2 \pm 8.5$ years; range 30 to 55 years) as control group. The sexual function of the participants was assessed by 19 -item FSFI.

Results: Mean duration of pSS in the patient group was 35.9 months (range 3 to 264 months). Significantly higher number of pSS patients reported positive history for vaginal infection compared to controls $(n=26,83.9 \%$ vs $n=7,25.9 \%$, respectively; $p<0.001)$ without any difference in endocervical culture result. Cervical smear assessment revealed more inflammation and atrophy in patient group compared to control group ( $p=0.001)$. Mean FSFI total score was significantly lower in patient group than control group ( $18.9 \pm 9.9 \mathrm{vs} 25.1 \pm 5.1$, respectively; $\mathrm{p}=0.004)$. Similarly, five out of six domains of FSFI -arousal, lubrication, orgasm, satisfaction, and pain- were significantly lower in patient group. FSFI total and subscale scores, except for pain, were found to be negatively correlated with duration of pSS.

Conclusion: The pSS causes sexual dysfunction in female patients. Furthermore, as disease duration increases, female sexual function decreases. Clinical management of female patients with pSS should cover the assessment of their sexual functionality and taking the necessary precautions to maintain satisfactory quality of life and treatment outcome.

Keywords: Dyspareunia; sexual function; Sjögren's syndrome.
\end{abstract}

Primary Sjögren's syndrome (pSS) is an autoimmune, chronic-systemic disease determined by both functional and structural abnormalities of exocrine glands throughout the body. ${ }^{1-5}$ The annual incidence of the disease in the literature is 3.9-5.3 per $100,000.6$ Two studies from Turkey estimated the prevalence ranging from $0.21 \%$ to $0.72 \%$ depending on the classification criteria. ${ }^{7,8}$ pSS occurs 10 to 20 times more frequently in females over males. ${ }^{9,10}$

Sjögren's syndrome-induced abnormalities of exocrine glands primarily lead to dryness of eyes and mouth. Clinical presentation may vary from sicca symptoms of dry eyes, dry mouth to severe systemic symptoms. ${ }^{6}$ In addition to these, female patients with pSS often were reported to have vaginal dryness and consequent chronic dyspareunia. ${ }^{11,12}$ Sometimes, chronic dyspareunia may be the presenting symptom in yet undiagnosed pSS cases. ${ }^{13}$ However, most females diagnosed with pSS are not informed about vaginal dryness, consequent chronic dyspareunia, and sexual problems associated with the condition. ${ }^{5}$ 
Although chronic dyspareunia is a well-reported symptom in females with pSS, its impact on sexual functionality of patients has rarely been studied. ${ }^{14}$ However, it is known that there is a possible relationship between sexual well-being and overall life satisfaction in females. ${ }^{15}$ In spite of this fact, female sexual functionality is a difficult subject to talk with patients, particularly in traditional societies like Turkey. Therefore, data on the impact of pSS on female sexual functionality need to be increased to identify the effect of this chronic disease on life of women and to develop management strategies against sexual dysfunction. Therefore, in this study, we aimed to evaluate the sexual function of female patients with pSS in comparison to healthy females by using the Female Sexual Function Index (FSFI).

\section{PATIENTS AND METHODS}

This case-control study included a total of 31 female patients (mean age 46.0 \pm 10.2 years; range 30 to 68 years) who had been under follow-up with diagnosis of pSS according to the American-European Consensus Group classification criteria ${ }^{16}$ and expert rheumatologist opinion in Cumhuriyet University Medical Faculty between June 2014 and June 2015, and 27 healthy females (mean age $44.2 \pm 8.5$ years; range 30 to 55 years) as control group. The healthy subjects were selected among hospital staff of the same ethnicity as the patients. The participants of this study had a sexual partner.

Exclusion criteria were as follows: chronic renal or hepatic impairment, presence of malignancy, smoking, other rheumatic/autoimmune disorders, medication or hormones having a potential to affect sexual functions, and history of gynecologic surgery within the past three months.

The study protocol was approved by the Institutional Ethics Committee (date 13.05.2014, no 2014-05/03) and conducted in accordance with the principles of the Helsinki Declaration and local regulations. All study participants gave written informed consents before any studyrelated procedures.

The following data were recorded for each study participant: sociodemographic factors, smoking status, duration of pSS (from diagnosis) and treatments applied, concomitant diseases, fertility history including postmenopausal status, gravidity, parity, vaginal infection, endocervical culture, and cervical smear. The cervical smear findings were evaluated by a pathologist and reported semi-quantitatively using the neutrophillymphocyte percentage.

Sexual function of the participants was assessed by 19-item FSFI. The FSFI evaluates sexual function in six subdomains: arousal, desire, lubrication, orgasm, pain, satisfaction. Higher scores demonstrate preferable sexual function. The total score is obtained by adding the scores of six-subdomains having a range of $2-36 .{ }^{17}$ The validity and reliability of Turkish version of FSFI have been shown by Öksüz and Malhan. ${ }^{18}$

The primary outcome of the study was FSFI total and subscale scores in patient group in comparison to control group. The secondary outcome was the correlation between duration of pSS and FSFI scores of patients.

\section{Statistical analysis}

Study data were first analyzed by descriptive statistics (e.g., mean, standard deviation, range, frequency, and percentage). Parameters of patient and control groups were compared using the Student's $t$ test for continuous variables and Mann-Whitney U test for non-parametric data (FSFI and disease duration) and chi-square test for categorical variables. Pearson correlation coefficient was calculated to measure strength of the linear relationship between duration of pSS and FSFI scores. Logistic regression analysis was performed for confounders (i.e. age). Statistical analysis was performed with IBM SPSS version 22.0 (IBM Corp., Armonk, NY. USA). Statistical level of significance was set to $p<0.05$.

\section{RESULTS}

Mean duration of pSS in the patient group was 35.9 months (range 3 to 264 months). The most commonly used agent for the treatment of pSS was hydroxychloroquine, alone or in combination with prednisone $(n=20,64.5 \%)$. pSS patients did not show significant differences from healthy controls in terms of education level, smoking status, or comorbidities ( $p>0.05$ for all, Table 1). 
Table 1. Sociodemographic and clinical findings of patient and control groups

\begin{tabular}{|c|c|c|c|c|c|c|c|c|c|}
\hline & \multicolumn{4}{|c|}{ Patients with pSS $(n=31)$} & \multicolumn{4}{|c|}{ Healthy controls $(n=27)$} & \multirow[b]{2}{*}{$p$} \\
\hline & $\mathrm{n}$ & $\%$ & Mean \pm SD & Min-Max & $\mathrm{n}$ & $\%$ & Mean \pm SD & $\operatorname{Min}-\operatorname{Max}$ & \\
\hline Age (year) & & & $46.0 \pm 10.2$ & $30-68$ & & & $44.2 \pm 8.5$ & $30-55$ & 0.087 \\
\hline Educational level & & & & & & & & & 0.289 \\
\hline Low (primary-middle school) & 11 & 35.5 & & & 5 & 18.5 & & & \\
\hline Medium (high school) & 17 & 54.8 & & & 17 & 63.0 & & & \\
\hline High (university or higher education) & 3 & 9.7 & & & 5 & 18.5 & & & \\
\hline Smoking status & 8 & 25.8 & & & 4 & 14.8 & & & 0.303 \\
\hline Sjögren's syndrome duration (month) & & & $35.9 \pm 47.8$ & $3-264$ & & & $\mathrm{~N} / \mathrm{A}$ & & $\mathrm{N} / \mathrm{A}$ \\
\hline Sjögren's syndrome treatment & & & & & & & & & N/A \\
\hline Hydroxychloroquine & 12 & 38.7 & & & & & N/A & & \\
\hline Hydroxychloroquine + prednisone & 8 & 25.8 & & & & & N/A & & \\
\hline Prednisone & 3 & 9.7 & & & & & N/A & & \\
\hline Disease-modifying antirheumatic drugs & 1 & 3.2 & & & & & N/A & & \\
\hline Hydroxychloroquine + prednisone + & & & & & & & & & \\
\hline disease-modifying antirheumatic drugs & 6 & 19.4 & & & & & N/A & & \\
\hline Biologic agents & 1 & 3.2 & & & & & N/A & & \\
\hline Comorbidity & & & & & & & & & 0.177 \\
\hline None & 21 & 67.7 & & & 19 & 70.4 & & & \\
\hline Hypertension & 1 & 3.2 & & & 3 & 11.1 & & & \\
\hline Diabetes mellitus & 0 & 0 & & & 2 & 7.4 & & & \\
\hline Hyperthyroidism & 1 & 3.2 & & & 2 & 7.4 & & & \\
\hline Hypertension + diabetes mellitus & 1 & 3.2 & & & 1 & 3.7 & & & \\
\hline Rheumatoid arthritis & 4 & 12.9 & & & 0 & 0 & & & \\
\hline Raynaud's syndrome & 2 & 6.5 & & & 0 & 0 & & & \\
\hline Familial Mediterranean fever & 1 & 3.2 & & & 0 & 0 & & & \\
\hline
\end{tabular}

Number of postmenopausal females in patient and control groups did not differ significantly $(n=7,22.6 \%$ vs $n=5, \quad 18.5 \%$, respectively; $p=0.703$ ). However, gravidity and parity rates were significantly higher in patient group (Table 2). Significantly more females in patient group reported positive history for vaginal infection ( $\mathrm{n}=26,83.9 \%$ vs $\mathrm{n}=7,25.9 \%$, respectively; $\mathrm{p}<0.001)$ without any difference in endocervical culture result (Table 2). Cervical smear assessment revealed higher inflammation and atrophy rates in patient group compared to control group ( $p=0.001$, Table 2).

Mean FSFI total score was significantly lower in patient group than control group $(18.9 \pm 9.9$ vs $25.1 \pm 5.1$, respectively; $p=0.004)$. Similarly, five out of six domains of FSFI -arousal, lubrication, orgasm, satisfaction, and pain- were significantly lower in patient group (Table 3).

In the correlation analysis, FSFI total and subscale scores, except for pain, were found to be

Table 2. Fertility history of patient and control groups

\begin{tabular}{|c|c|c|c|c|c|c|c|c|c|}
\hline & \multicolumn{4}{|c|}{ Patients with pSS $(n=31)$} & \multicolumn{4}{|c|}{ Healthy controls $(\mathrm{n}=27)$} & \multirow[b]{2}{*}{$p$} \\
\hline & $\mathrm{n}$ & $\%$ & Mean \pm SD & Min-Max & $\mathrm{n}$ & $\%$ & Mean \pm SD & Min-Max & \\
\hline Postmenopausal status & 7 & 22.6 & & & 5 & 18.5 & & & 0.703 \\
\hline Gravidity & & & $5.1 \pm 3.9$ & $0-12$ & & & $2.6 \pm 1.6$ & $0-6$ & 0.002 \\
\hline Parity & & & $3.7 \pm 3.2$ & $0-8$ & & & $2.2 \pm 1.1$ & $0-5$ & 0.018 \\
\hline History of vaginal infection & 26 & 83.9 & & & 7 & 25.9 & & & $<0.001$ \\
\hline Endocervical culture & & & & & & & & & 0.635 \\
\hline Negative & 2 & 6.5 & & & 1 & 12.5 & & & \\
\hline Lactobacilli & 12 & 38.7 & & & 2 & 25 & & & \\
\hline Escherichia coli & 1 & 3.2 & & & 1 & 12.5 & & & \\
\hline Enterococcus faecalis & 9 & 29 & & & 1 & 12.5 & & & \\
\hline Candida albicans & 5 & 16.1 & & & 3 & 37.5 & & & \\
\hline Streptococcus agalactiae & 1 & 3.2 & & & 0 & 0 & & & \\
\hline Gardnerella vaginalis & 1 & 3.2 & & & 0 & 0 & & & \\
\hline Cervical smear & & & & & & & & & 0.001 \\
\hline Benign cellular changes, no inflammation & 7 & 22.6 & & & 19 & 70.4 & & & \\
\hline Benign cellular changes, inflammation & 19 & 61.3 & & & 8 & 29.6 & & & \\
\hline Benign cellular changes, atrophy & 5 & 16.1 & & & 0 & 0 & & & \\
\hline
\end{tabular}




\begin{tabular}{|c|c|c|c|c|c|}
\hline & \multicolumn{2}{|c|}{ Patients with pSS $(n=31)$} & \multicolumn{2}{|c|}{ Healthy controls $(n=27)$} & \multirow[b]{2}{*}{$p$} \\
\hline & Mean \pm SD & Min-Max & Mean \pm SD & Min-Max & \\
\hline FSFI total (2-36) & $18.9 \pm 9.9$ & $2-34.6$ & $25.1 \pm 5.1$ & $14.4-32.9$ & 0.004 \\
\hline \multicolumn{6}{|l|}{ FSFI subscale score } \\
\hline Desire (1.2-6) & $2.6 \pm 1.3$ & $1.2-4.8$ & $3.2 \pm 1$ & $1.6-6$ & 0.059 \\
\hline Arousal (0-6) & $2.8 \pm 1.8$ & $0-6$ & $3.9 \pm 1.1$ & $1.2-5.7$ & 0.008 \\
\hline Lubrication (0-6) & $3.4 \pm 2$ & $0-6$ & $4.8 \pm 1.1$ & $2.4-6$ & 0.002 \\
\hline Orgasm (0-6) & $3.2 \pm 2$ & $0-6$ & $4.3 \pm 1.1$ & $1.6-6$ & 0.013 \\
\hline Satisfaction (0.8-6) & $3.5 \pm 1.7$ & $0-6$ & $4.4 \pm 1$ & $2.4-6$ & 0.023 \\
\hline Pain $(0-6)$ & $3.3 \pm 2$ & $0-6$ & $4.6 \pm 1.4$ & $1.2-6$ & 0.008 \\
\hline
\end{tabular}

negatively correlated with duration of pSS; in other words, as disease duration increased, FSFI scores showing female sexual function decreased (Table 4).

\section{DISCUSSION}

In this case-control study, we showed that females with pSS have significant sexual dysfunction in comparison to healthy females with varying severity depending on the duration of disease. Our study is one of the limited number of studies in the literature on the impact of pSS on the female sexual functionality assessed by a validated self-report measure, FSFI.

Systemic autoimmune diseases including pSS affect various organs and systems in the body leading to dysfunction in such organs and systems, with sexual dysfunction being one of the consequences. ${ }^{19,20}$ Sexual dysfunction in females with systemic autoimmune diseases is most probably multifactorial due to the chronicity,

Table 4. Spearman correlation coefficient for relationship between duration of primary Sjögren's syndrome and Female Sexual Function Index scores

\begin{tabular}{lcr}
\hline & \multicolumn{2}{c}{ Duration of pSS } \\
\cline { 2 - 3 } & $\mathrm{r}$ & $\mathrm{p}$ \\
\hline FSFI total score & -0.290 & 0.029 \\
FSFI subscale scores & & \\
$\quad$ Desire & -0.322 & 0.015 \\
$\quad$ Arousal & -0.304 & 0.022 \\
Lubrication & -0.323 & 0.014 \\
Orgasm & -0.283 & 0.033 \\
Satisfaction & -0.318 & 0.016 \\
Pain & -0.042 & 0.760 \\
\hline pSS: Primary Sjögren's syndrome; r: Correlation coefficient. Spearman \\
correlation test was used; FSFI: Female Sexual Function Index. \\
\multicolumn{2}{l}{} \\
\multicolumn{2}{l}{}
\end{tabular}

pathophysiology, activity, and psychological impact of disease, and drugs used for the treatment.19,20 Among these factors, vaginal discomfort and pain may mostly contribute to sexual dysfunction in patients with pSS. ${ }^{20}$

Although recent immunologic and clinical researches led to significant advances in diagnosis and management of pSS, there is rather a small number of studies on the impact of the condition on sexual functionality of females. ${ }^{1,2,21}$ van Nimwegen et al. ${ }^{14}$ recently studied the impact of pSS on female sexual function by a number of self-administered questionnaires including FSFI on 46 patients and 43 age-matched controls and found that females with pSS have disrupted sexual function and more sexual discomfort compared with healthy controls. In the mentioned study, ${ }^{14}$ median total FSFI scores were 20.6 and 30.3 for females with and without pSS, respectively. They also found that sexual function is affected by physical factors such as vaginal dryness, pain and fatigue, and psychological involvement of pSS. Similarly, in the present study, we found mean FSFI total scores as 18.9 and 25 in patients with pSS and age-matched healthy females, respectively $(p=0.004)$, showing the impairment of sexual functionality in females with pSS. Furthermore, as in the study by van Nimwegen et al., ${ }^{14}$ five out of six domains of FSFI -arousal, lubrication, orgasm, satisfaction, and pain- were significantly lower in patients with pSS in our study. In the study by van Nimwegen et al., ${ }^{14}$ all subscale scores were significantly lower in females with pSS except for satisfaction score.

An important finding of the present study was that sexual functionality of patients with pSS depended on the duration of disease. In other 
words, as duration of pSS increased, female sexual functionality decreased.

Disease onset following menopause is a common occurrence in pSS patients. ${ }^{22}$ However, in our study, there was no difference between pSS patients and controls in terms of rate of postmenopausal females. Previous studies reported no difference in fertility, parity or reproductive success rates between pSS patients and controls. ${ }^{12}$ However, in the present study, gravidity and parity rates were significantly higher in patients with $\mathrm{pSS}$.

We also found more inflammation and atrophy in cervical smear in patients with pSS than controls, most probably due to vaginal dryness in the patients. Vaginal dryness and dyspareunia are common symptoms of females affected with pSS. ${ }^{23}$ These gynecological symptoms lead to impaired sexual intercourse, negatively affecting pleasure, satisfaction, and sexual ability. ${ }^{24}$ As in our study, Priori et al. ${ }^{25}$ showed that pSS (pre- and postmenopausal) patients had impaired quality of sexual function, and FSFI was found as a valuable measure for assessing sexual function. Furthermore, Cirpan et al. ${ }^{26}$ performed cervical cytology, colposcopic examination, and human papillomavirus deoxyribonucleic acid testing in females with Sjögren's syndrome, and demonstrated similar findings in Sjögren's syndrome patients and controls. However, our study showed that pSS patients had higher prevalence of inflammation and atrophy in cervical smear.

The main limitations of our study were the small sample size and cross-sectional study design that prevented us from reaching a definitive conclusion on the prevalence of sexual dysfunction among females with pSS. However, this is one of the few studies in the literature on the clinical effect of pSS on female sexual functionality, the importance of which has been well-established for females to maintain a satisfactory quality of life. ${ }^{15,19,20}$

In conclusion, pSS causes sexual dysfunction in females with a severity increasing with disease duration. Clinical management of females with pSS should cover the assessment of sexual functionality of patients and taking the necessary precautions to maintain satisfactory quality of life and treatment outcome. Considering the importance of sexual functionality in the overall health of females, both patients and physicians should be informed on the possibility of pSS-related female sexual dysfunction. The findings of this study should be confirmed by further large-scale prospective studies on the prevalence, impact on patients' quality of life, diagnosis and treatment approaches of sexual dysfunction among females with pSS. Also, further studies are required on the management of discomfort caused by sexual dysfunction in female pSS patients.

\section{Declaration of conflicting interests}

The authors declared no conflicts of interest with respect to the authorship and/or publication of this article.

\section{Funding}

The authors received no financial support for the research and/or authorship of this article.

\section{REFERENCES}

1. Luciano N, Valentini V, Calabrò A, Elefante E, Vitale A, Baldini C, et al. One year in review 2015: Sjögren's syndrome. Clin Exp Rheumatol 2015;33:259-71.

2. Maslinska M, Przygodzka M, Kwiatkowska B, Sikorska-Siudek K. Sjögren's syndrome: still not fully understood disease. Rheumatol Int 2015;35:233-41.

3. Cornec D, Jamin C, Pers JO. Sjögren's syndrome: where do we stand, and where shall we go? J Autoimmun 2014;51:109-14.

4. Konttinen YT, Porola P, Konttinen L, Laine M, Poduval P. Immunohistopathology of Sjögren's syndrome. Autoimmun Rev 2006;6:16-20.

5. Schoofs N. Caring for women living with Sjögren's syndrome. J Obstet Gynecol Neonatal Nurs 2003;32:589-93.

6. Patel R, Shahane A. The epidemiology of Sjögren's syndrome. Clin Epidemiol 2014;6:247-55.

7. Birlik M, Akar S, Gurler O, Sari I, Birlik B, Sarioglu S, et al. Prevalence of primary Sjogren's syndrome in Turkey: a population-based epidemiological study. Int J Clin Pract 2009;63:954-61.

8. Kabasakal Y, Kitapcioglu G, Turk T, Oder G, Durusoy R, Mete N, et al. The prevalence of Sjögren's syndrome in adult women. Scand $J$ Rheumatol 2006;35:379-83.

9. Brandt JE, Priori R, Valesini G, Fairweather D. Sex differences in Sjögren's syndrome: a comprehensive review of immune mechanisms. Biol Sex Differ 2015;6:19. 
10. Reksten TR, Jonsson MV. Sjögren's syndrome: an update on epidemiology and current insights on pathophysiology. Oral Maxillofac Surg Clin North Am 2014;26:1-12.

11. Lehrer S, Bogursky E, Yemini M, Kase NG, Birkenfeld A. Gynecologic manifestations of Sjögren's syndrome. Am J Obstet Gynecol 1994;170:835-7.

12. Skopouli FN, Papanikolaou S, Malamou-Mitsi V, Papanikolaou N, Moutsopoulos HM. Obstetric and gynaecological profile in patients with primary Sjögren's syndrome. Ann Rheum Dis 1994;53:569-73.

13. Mulherin DM, Sheeran TP, Kumararatne DS, Speculand B, Luesley D, Situnayake RD. Sjögren's syndrome in women presenting with chronic dyspareunia. Br J Obstet Gynaecol 1997;104:1019-23.

14. van Nimwegen JF, Arends S, van Zuiden GS, Vissink A, Kroese FG, Bootsma H. The impact of primary Sjögren's syndrome on female sexual function. Rheumatology (Oxford) 2015;54:1286-93.

15. Stephenson KR, Meston CM. The conditional importance of sex: exploring the association between sexual well-being and life satisfaction. J Sex Marital Ther 2015;41:25-38.

16. Vitali C, Bombardieri S, Jonsson R, Moutsopoulos HM, Alexander EL, Carsons SE, et al. Classification criteria for Sjögren's syndrome: a revised version of the European criteria proposed by the AmericanEuropean Consensus Group. Ann Rheum Dis 2002;61:554-8.

17. Rosen R, Brown C, Heiman J, Leiblum S, Meston C, Shabsigh R, et al. The Female Sexual Function Index (FSFI): a multidimensional self-report instrument for the assessment of female sexual function. J Sex Marital Ther 2000;26:191-208.
18. Öksüz E, Malhan S (2005) The validity and reliability of Turkish version of Female Sexual Function IndexFSFI Sendrom 2005;17:54-60.

19. Araujo DB, Borba EF, Abdo $\mathrm{CH}$, Souza Lde A, Goldenstein-Schainberg C, Chahade WH, et al. Sexual function in rheumatic diseases. Acta Reumatol Port 2010;35:16-23. [Abstract]

20. Tristano AG. The impact of rheumatic diseases on sexual function. Rheumatol Int 2009;29:853-60.

21. Sada PR, Isenberg D, Ciurtin C. Biologic treatment in Sjögren's syndrome. Rheumatology (Oxford) 2015;54:219-30.

22. Mavragani CP, Fragoulis GE, Moutsopoulos HM. Endocrine alterations in primary Sjogren's syndrome: an overview. J Autoimmun 2012;39:354-8.

23. Marchesoni D, Mozzanega $\mathrm{B}$, De Sandre $\mathrm{P}$, Romagnolo C, Gambari PF, Maggino T. Gynaecological aspects of primary Sjogren's syndrome. Eur J Obstet Gynecol Reprod Biol 1995;63:49-53.

24. Maddali Bongi S, Del Rosso A, Orlandi M, Matucci-Cerinic M. Gynaecological symptoms and sexual disability in women with primary Sjögren's syndrome and sicca syndrome. Clin Exp Rheumatol 2013;31:683-90.

25. Priori R, Minniti A, Derme M, Antonazzo B, Brancatisano F, Ghirini S, et al. Quality of Sexual Life in Women with Primary Sjögren Syndrome. J Rheumatol 2015;42:1427-31.

26. Cirpan T, Guliyeva A, Onder G, Terek MC, Ozsaran A, Kabasakal Y, et al. Comparison of human papillomavirus testing and cervical cytology with colposcopic examination and biopsy in cervical cancer screening in a cohort of patients with Sjogren's syndrome. Eur J Gynaecol Oncol 2007;28:302-6. 